STUDI

FRANCESI

\section{Studi Francesi}

Rivista quadrimestrale fondata da Franco Simone

190 (LXIV | I) | 2020

Varia - fasc. I - gennaio-aprile 2020

\title{
Medieval Francophone Literary Culture Outside France. Studies in the Moving Word, sous la direction de N. MORATO et D. SCHOENAERS
}

\section{Elisabetta Barale}

\section{(2) OpenEdition}

Journals

\section{Édition électronique}

URL : https://journals.openedition.org/studifrancesi/22137

DOI : $10.4000 /$ studifrancesi.22137

ISSN : 2421-5856

Éditeur

Rosenberg \& Sellier

\section{Édition imprimée}

Date de publication : 1 avril 2020

Pagination : 139-140

ISSN : 0039-2944

\section{Référence électronique}

Elisabetta Barale, « Medieval Francophone Literary Culture Outside France. Studies in the Moving Word, sous la direction de N. morato et D. schoenaers », Studi Francesi [En ligne], 190 (LXIV | I) | 2020, mis en ligne le 01 avril 2020, consulté le 03 août 2021. URL : http://journals.openedition.org/studifrancesi/ 22137 ; DOI : https://doi.org/10.4000/studifrancesi.22137

Ce document a été généré automatiquement le 3 août 2021

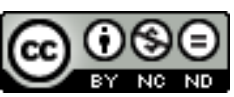

Studi Francesi è distribuita con Licenza Creative Commons Attribuzione - Non commerciale - Non opere derivate 4.0 Internazionale. 


\title{
Medieval Francophone Literary Culture Outside France. Studies in the Moving Word, sous la direction de N. MORATO et D. SCHOENAERS
}

\author{
Elisabetta Barale
}

\section{RÉFÉRENCE}

Medieval Francophone Literary Culture Outside France. Studies in the Moving Word, sous la direction de N. MORATO et D. SCHOENAERS, Turnhout, Brepols, 2018, «Medieval Texts and Cultures of Northern Europe» 28, XV + 571 pp.

1 Ce beau volume consacré à la diffusion de la littérature française médiévale en dehors de la France constitue l'un des résultats du projet AHRC Medieval Francophone Literary Culture Oustide France (MFLCOF, 2011-2015), dirigé par Simon GAUNT (King's College London), Jane GILBERT (University College London) et Bill BURGWINKLE (University of Cambridge). Il réunit dix-neuf contributions, réparties en cinq sections reprenant les lignes de force du projet.

2 Après une introduction générale rédigée par Nicola MORATO et Dirk SCHOENAERS (Introduction, pp. 1-28), la première section (Southern Axis: Mediterranean Europe and the Middle East, pp. 29-210) fait le point sur la circulation des œuvres littéraires françaises dans quelques pays de la Méditerranée: Italie, Espagne, Chypre.

3 Fabio ZINELLI (Inside/Outside Grammar: The French of Italy between Structuralism and Trends of Exoticism, pp. 31-72) passe en revue les stratégies méthodologiques adoptées par la critique internationale dans l'étude de la langue et de la littérature franco-italienne; ensuite, il fait appel à la notion de «porosité» pour décrire les phénomènes 
linguistiques qui intéressent les traductions réalisées à Gênes et à Pise, car elles présentent plusieurs mots hybrides issus du français.

Charmaine LEE (That Obscure Object of Desire: French in Southern Italy, pp. 73-100) s'arrête sur l'emploi du français dans l'Italie du Sud. Elle retrace l'histoire de la période normande et angevine, en soulignant que c'est grâce à l'arrivée de Charles I ${ }^{\text {er }} \mathrm{d}^{\prime}$ Anjou qu'on assista au développement d'un milieu culturel français. Après avoir énuméré les textes sortis des ateliers napolitains, elle en effectue une analyse linguistique visant à identifier les italianismes et les formes typiques de la scripta d'Outremer.

F. Regina PSAKI (Medieval Misogyny and the French of Italy: the "Chastiemusart" and the "Proverbia que dicuntur super natura feminarum", pp. 101-139) illustre le rapport entre le Chastiemusart et les Proverbia que dicuntur super natura feminarum, une réécriture italienne rédigée dans la première moitié $d u \mathrm{xv}^{\mathrm{e}}$ siècle et transmise par le codex unique Berlin, Staatsbibliothek, Hamilton 390. Elle réfléchit sur le rôle joué par la voix narrative dans les deux œuvres et sur la mise en image de l'intrigue dans les Proverbia, en concluant que les textes misogynes français inspiraient des adaptations créatives dans l'Italie du Nord.

6 Les manuscrits du Lancelot en prose conservés dans les bibliothèques espagnoles sont examinés par Lourdes SORIANO ROBLES (French Manuscripts of the "Lancelot en prose" Preserved in Spain and Portugal, pp.141-164). Sur la base d'une étude codicologique et iconographique, l'A. montre que ni les fragments de Puigcerdà, ni les volumes de Madrid et de l'Escorial ne furent confectionnés en France; au contraire, le codex de Madrid serait issu de la cour d'Aragon, tandis que les autres se rattacheraient au groupe des manuscrits copiés à Gênes.

7 Chiara CONCINA (Boethius in Cyprus? Pierre de Paris's Translation of the "Consolatio Philosophiae", pp. 165-190) s'interroge sur la provenance du codex Città del Vaticano, $\mathrm{BAV}$, Vat. lat. 4788, transmettant la traduction en prose et le commentaire du De consolatione Philosophiae par Pierre de Paris. Après avoir avancé deux hypothèses - le manuscrit aurait été copié en Chypre dans un atelier italien ou bien il aurait été réalisé sur le continent d'après un modèle d'Outremer - elle affirme qu'à l'état actuel de la recherche, face aux évidences linguistiques, iconographiques et littéraires témoignant d'une osmose culturelle entre Orient et Occident, la question demeure ouverte.

8 Vraisemblablement composé en Chypre en 1228, la Disme de Penitanche du chevalier artésien Jehan de Journi fait l'objet de l'étude de Marisa GALVEz (Jehan de Journi's “Disme de Penitanche" and the Production of a Vernacular Confessional Text in Outremer, pp. 191-210). L'A. se focalise sur l'emploi du mot «franchise» et observe un glissement sémantique par rapport aux occurrences attestées dans les œuvres littéraires occidentales; influencé par le milieu culturel d'Outremer, ce long poème confessionnel destiné à un public laïque redéfinit la notion de «franchise» en tant qu'invitation aux contributions financières et aux actions militaires.

9 La deuxième section (Northern Axis: England, the Low Countries and the Empire, pp. 211-319) envisage la circulation des œuvres littéraires françaises dans deux régions germanophones, l'Allemagne et les Pays-Bas.

10 Adrian STEvens (Wolfram von Eschenbach, Gottfried von Strassburg, and the Politics of Literary Adaptation: the Grail and Tristan Romances and the Court of Otto IV, 1198-1218, pp. 213-240) se penche sur la réception du Conte du Graal de Chrétien de Troyes et du Tristan de Thomas en Allemagne. Il suggère que tant le Parzival de Wolfram von Eschenbach que le Tristan 
de Gottfried von Strassburg auraient été adaptés pour l'entourage de l'empereur Otton IV. D'un côté, les reconstructions généalogiques de Wolfram permettent de relier le passé arthurien au passé historique de la cour allemande; de l'autre, l'adaptation de Gottfried ne manque pas de célébrer la lignée angevine du mécène.

La contribution de Frank BRANDSMA (Middle Dutch Poets and their Francophone Sources: Respect and Reservations, pp. 241-263) porte sur la diffusion de la littérature francophone dans les anciens Pays-Bas. Après avoir constaté que les premières traductions néerlandaises sont influencées par le prestige culturel du français, l'A. examine les travaux de quelques auteurs jugés «francophobes», comme Jacob van Maerlaent; il démontre qu'une prise de distance par rapport aux sources correspondrait au souhait d'affirmer l'indépendance linguistique et littéraire du néerlandais.

( Low Countries, pp. 265-287) étudie l'adaptation néerlandaise de La Mort le roi Artu (Artus doet), vraisemblablement réalisée par Lodewijk van Velthem en Brabant vers 1320 à partir d'une traduction flamande datant de 1280. Elle se concentre sur les interpolations attribuées au compilateur (le traité religieux du prologue, les prières, les récits historiques) témoignant d'un engagement personnel dans la réélaboration d'un texte de fiction critiqué par Jacob van Maerlaent.

Florent NoIRFAlise (Context-Based Compilation? The Use of the "Histoire ancienne jusqu'à César" and the Function of the "matière d'Alexandre" in the "Chronique de Baudouin d'Avesnes", pp. 289-319) propose un examen des sources de la première chronique universelle en français, la Chronique de Baudouin d'Avesnes, composée en Flandres vers 1270 , en concluant que le lectorat envisagé aurait été le même que celui des Alexanders Geesten de Jacob van Maerlaent.

La troisième (Material and Documentary Evidence, pp. 323-387) et la quatrième section (Textual Evidence, pp. 391-449) abordent les modalités de la transmission textuelle.

Puisque les notions de «porosité des frontières» et de «multilinguisme» ont fait l'objet d'un regain d'intérêt de la part de la communauté scientifique, Margriet HOOGVLIET (Religious Reading in French and Middle Dutch in the Southern Low Countries and Northern France, c. 1400 - c. 1520, pp. 323-347) présente les premiers résultats d'un projet en cours à l'Université de Gröningen concernant la circulation des textes religieux entre la France du Nord et les anciens Pays-Bas méridionaux; elle introduit notamment le concept de «communautés d'interprétation» pour désigner les groupes de lecteurs bilingues qui partageaient leur patrimoine libraire.

16 Victor JANTE (An Italian Reader of the "Chroniques d'Angleterre" by Jean de Wavrin: Pierre Villa and ms. BnF f. fr. 87, pp. 349-370) remarque que l'impact des Chroniques d'Angleterre de Jean de Wavrin sur le public de l'époque ne doit pas être sous-estimé. Il étudie le manuscrit Paris, BnF, fr. 87, réalisé à Bruges dans la seconde moitié du xve siècle pour le banquier d'origine piémontaise Pierre de Villa, et avance l'hypothèse que son commanditaire aurait entretenu des rapports commerciaux et diplomatiques avec l'Angleterre.

17 En adoptant une perspective différente par rapport à celle qui caractérise les travaux de ce volume, Eliza ZINGESSER (Francophone Troubadours: Assimilating Occitan Lyric in Medieval France, pp. 371-387) se penche sur la diffusion de la lyrique des troubadours en France. Elle remarque que les textes occitans sont soumis à des procédés de 
«francisation», dont le but principal consiste en l'adaptation des tournures linguistiques au système phonétique du français.

Inaugurant la quatrième section, Anna ALBERNI (Machaut's Literary Legacy in the Crown of Aragon: the Catalan Chansonnier Vega-Aguiló and the anonymous "Roman de Cardenois", pp. 391-410) expose les résultats partiels d'un projet européen mené à l'Université de Barcelone, dont l'un des objectifs prévoit la réalisation d'une nouvelle édition du Roman de Cardenois. L'A. observe que trois des douze insertions lyriques cachées dans le Roman se lisent aussi dans le chansonnier Vega-Aguiló; les citations de Machaut permettent surtout d'apprécier l'influence du poète sur l'entourage aragonais $\mathrm{du} \mathrm{Xv}^{\mathrm{e}}$ siècle.

La matière de Troie est au cœur des recherches de Marilynn DESMOND («Magna Graecia» and the Matter of Troy in the Francophone Mediterranean, pp. 411-431), qui entame une réflexion sur la notion de «Magna Graecia» à l'époque médiévale, en se focalisant sur la transmission et la traduction des textes à l'origine de la diffusion de l'histoire troyenne dans le bassin de la Méditerranée ("Roman de Troie" de Benoît de Sainte-Maure et "Histoire ancienne jusqu'à César").

L'Histoire ancienne jusqu'à César fait aussi l'objet de l'étude de Jessica SToll («Translatio» Networks in the Prose Troy Tradition, pp. 433-449), qui mène une comparaison entre la première et la seconde rédaction de l'œuvre dans laquelle le récit de la guerre de Troie, fondé sur le De Excidio Troiae Historia de Darès le Phrygien est remplacé par la cinquième mise en prose du Roman de Troie de Benoît de Sainte-Maure (Prose V).

21 La cinquième et dernière section (Constructing «auctoritas»: French, Occitan, and Italian, pp. 453-541) s'occupe enfin de réfléchir sur les moyens de création de l'autorité.

À travers l'étude parallèle de l'Historia Francorum d'Aguilers, des Gesta Tancredi de Ralph de Caen et de l'Historia Hierosolymitana de Foucher de Chartres, Patricia HARRIS STÄBLEIN GILLIES (Staging Francophone Identities: Latin First Crusade Narratives and the Epic Conflict of French and Occitan, pp. 453-472) situe l'émergence de la distinction identitaire entre les Francs et les Provençaux à l'époque de la première croisade, dans le contexte de la découverte de la Sainte Lance à Antioche.

Courtney Joseph weLLS («In lingua est diversitas»: Medieval Francophone and Occitanophone Literary Cultures in Catalonia and Italy, pp. 473-505) relit la Razos de trobar de Ramon Vidal et le De vulgari eloquentia de Dante pour montrer que les deux auteurs ne considèrent pas le français et l'occitan comme des langues nationales; au contraire, ils les présentent comme des instruments d'expression littéraire.

Laura CHUHAN CAMPBELL (French Literary Identity in Translation: the "Roman de la Rose" and its Tuscan Adaptations, pp.507-529) aborde enfin une forme particulière de translatio du français à l'italien: la transposition littéraire d'un texte d'un style à un autre. Elle analyse le Fiore et le Detto d'Amore, deux adaptations toscanes du Roman de la Rose, afin de démontrer que toute opération de réécriture comporte une négociation des aspects stylistiques de la source.

Précédés d'une conclusion rédigée par Bill BURGWINKLE (Afterword, pp. 531-541), l'index des manuscrits (pp. 543-546) et celui des noms (pp. 547-571) terminent le volume. 